\title{
REYNOLDS NUMBER EFFECT IN A PROBLEM OF SOUND GENERATION BY ROUND AND TRUNCATED CYLINDER STREAMLINED BY TURBULENT FLOW
}

\author{
V. Kopiev, M. Zaitsev, and N. Ostrikov \\ Central Aerohydrodynamic Institute (TsAGI) \\ Zhukovsky Str. 1, Zhukovsky, Moscow region 140180, Russia
}

\begin{abstract}
Well-known situation when bluff body is streamlining by turbulent flow is considered. Recently obtained experimental results for cylinder and role of small quadrupole sources in the wake are discussed. They appear to be strongly connected with the dipole sources located on the cylinder. Interference leads to surprising picture of the resulting sound field detected in experiment: equivalent dipole sources are located in the wake zone far downstream from the cylinder. The understanding of the effect of dipole abnormal shift helps to elaborate the modified cylinder (truncated cylinder) configurations which are considered. These configurations present attractive variant of airframe noise control where control realized by self-tuning of reflected signal leading to suppression of uncompensated dipoles.
\end{abstract}

\section{INTRODUCTION}

With continued success in engine noise reduction, airframe noise has emerged as a potentially significant contributor to overall acoustic emissions, particularly at landing conditions. The dominant sources of airframe noise are known to be associated with unsteadiness of separated and/or vortical flow regions around the high-lift system (i. e., flaps and slats) and the aircraft undercarriage (i.e., landing gear). Due to the complexity of three-dimensional (3D) vortices that may contribute to flow unsteadiness and the importance of surface geometry in scattering these vortical structures into sound, airframe noise is an extremely complex and challenging problem.

This is an Open Access article distributed under the terms of the Creative Commons Attribution-Noncommercial License 3.0, which permits unrestricted use, distribution, and reproduction in any noncommercial medium, provided the original work is properly cited. 


\section{RESULTS}

The well-known situation when circular cylinder is streamlining by turbulent flow is considered and new experimental results concerning process of sound generation are analyzed. Recent experiment $[1,2]$ which was carried out according to azimuthal decomposition technique (ADT) evidently demonstrated the fictitious shift of dipole sound source into the region far downstream from the cylinder (Fig. 1). The theoretical and numerical analysis [3] of the effect of abnormal shift showed that the dipole radiation in the frequency range considered is exactly the quadrupoles reflection from the curvilinear surface of the cylinder. They appear to be strongly connected with the dipole sources located on the cylinder. Interference leads to surprising picture of the resulting sound field: equivalent dipole sources are located in the separation zone far downstream from the cylinder. This effect manifests a dual role of small quadrupole sources in the low-frequency range: (i) quadrupoles located closely to the cylindrical surface create the dipole sources in the cylinder (which radiate the main noise); and ( $i i)$ quadrupoles displace the dipole source far downstream due to the interference. So, even for small Mach number, these quadrupole sources must be considered for correct noise prediction and mechanisms revealing.

On the basis of such understanding of the noise generation mechanism, the special configurations which lead to considerable noise suppression were proposed. When the curvature of the hard surface, from which the quadrupole source is reflected, decreases, the dipole noise component reduces (and disappears completely for the plane surface of reflection). Hence, if the rear of the element of cylindrical form, operating in the turbulent flow were cut off (flat-end cylinder), the noncompensated dipole component would decrease [2]. Since the cylinder surface curvature variations took place behind the line of the flow sep-

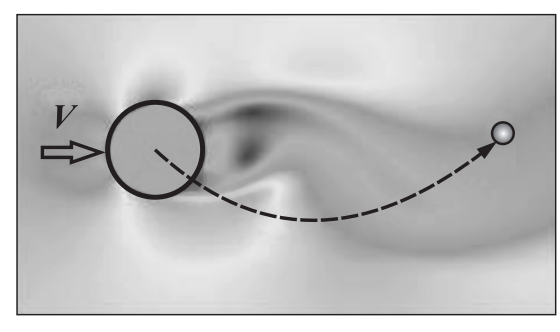

(a)

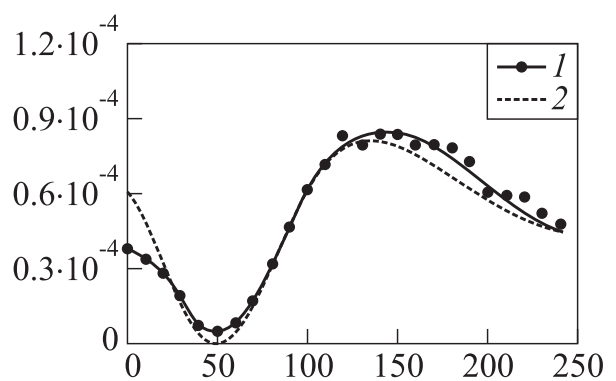

(b)

Figure 1 Experimental fictitious shift of dipole source downstream the streamlining cylinder: 1 - frequency range 1050-1350 Hz; and 2 - theoretical dipole, $x=50 \mathrm{~cm}$ 


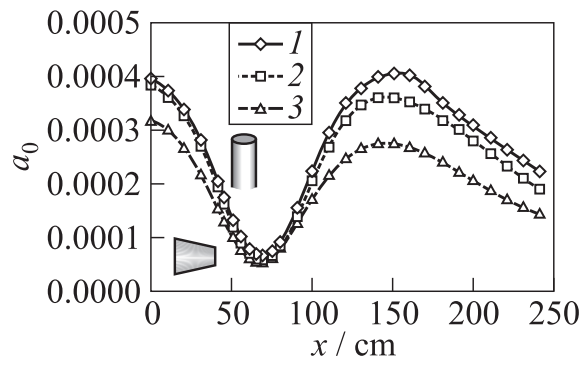

(a)

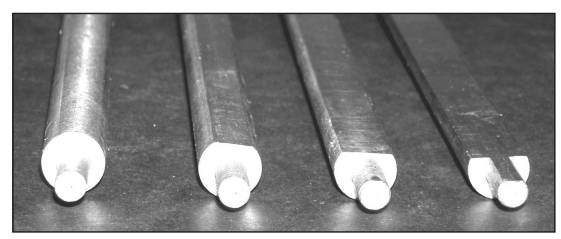

(b)

Figure 2 Noise suppression for truncated cylinders, $a_{0}(1$ - round cylinder, 2 small truncation, and 3 - middle truncation, frequency range $350-3200 \mathrm{~Hz})(a)$ and round and flat-end (truncated) cylinders $(b)$

aration, the indicated effect was achieved without considerable flow parameter variations and, hence, without deterioration of other aerodynamic characteristics. This not evident conclusion is supported by a series of experiments with small-scale models (Fig. 2b) carried out in anechoic chamber. It was established that for the cylinder (landing gear strut or engine stator rack) with a plane rear surface, the noise level in wide frequency band decreases by the value up to $5 \mathrm{~dB}$ over the whole range of observation angles [2,3] (Figs. $2 a$ and $3 c$ ).

Some new analytical results are considered to understand more carefully the mechanism of noise suppression by truncated cylinder (Fig. $3 a$ ), using the conform mapping of truncated form to circular one. It appears that truncated surface could play twofold role: when the sources are located closely to the middle of truncated part it will act as a noise suppressor. If the sources are located closely to the sharp corner (curvature is much more) the noise is increased considerably and truncated part acts as an amplifier. Turbulence characteristics in the wake and separation zone are measured by hot-wire anemometry and PIV (Fig. 4).

To uncover the effects of Reynolds number, the cylinders of different diameters including large-scale models $(3,12,40$, and $100 \mathrm{~mm})$ inserted in subsonic jet were tested in the anechoic chamber of TsAGI. The experimental database obtained in the current tests can provide the data for verification of theoreti- 


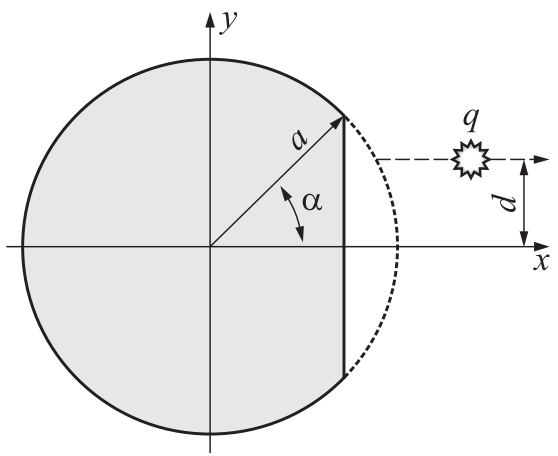

(a)

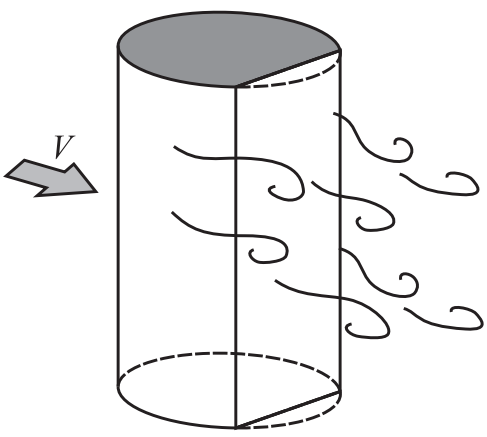

(b)

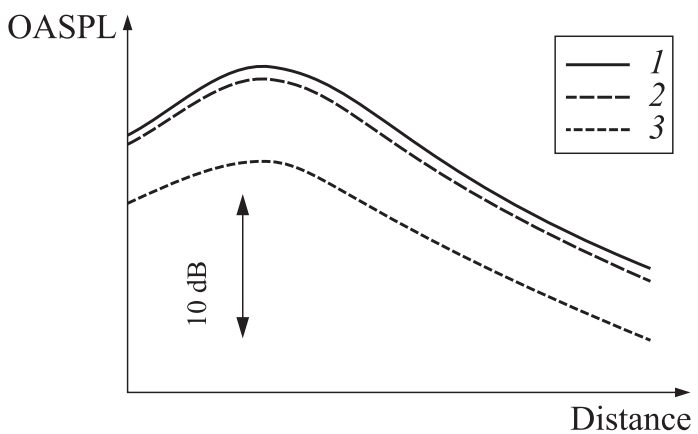

(c)

Figure 3 Circular cylinder truncated at the angle $\alpha(a)$, and noise measurements for round and flat-end cylinders: $(b)$ configuration; and $(c)$ overall averaged sound pressure level (OASPL) as a function of a distance along the jet axis demonstrating noise suppression for small-scale truncated cylinder

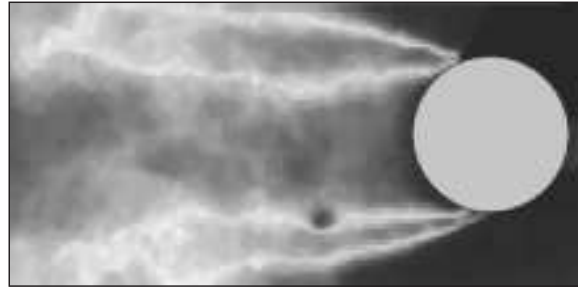

(a)

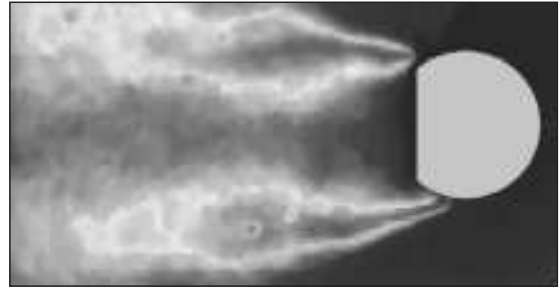

(b)

Figure 4 The PIV measurements: comparison of averaged vorticity distribution for circular $(a)$ and truncated $(b)$ geometries, which demonstrates identical properties of turbulence behind the round and truncated cylinders 

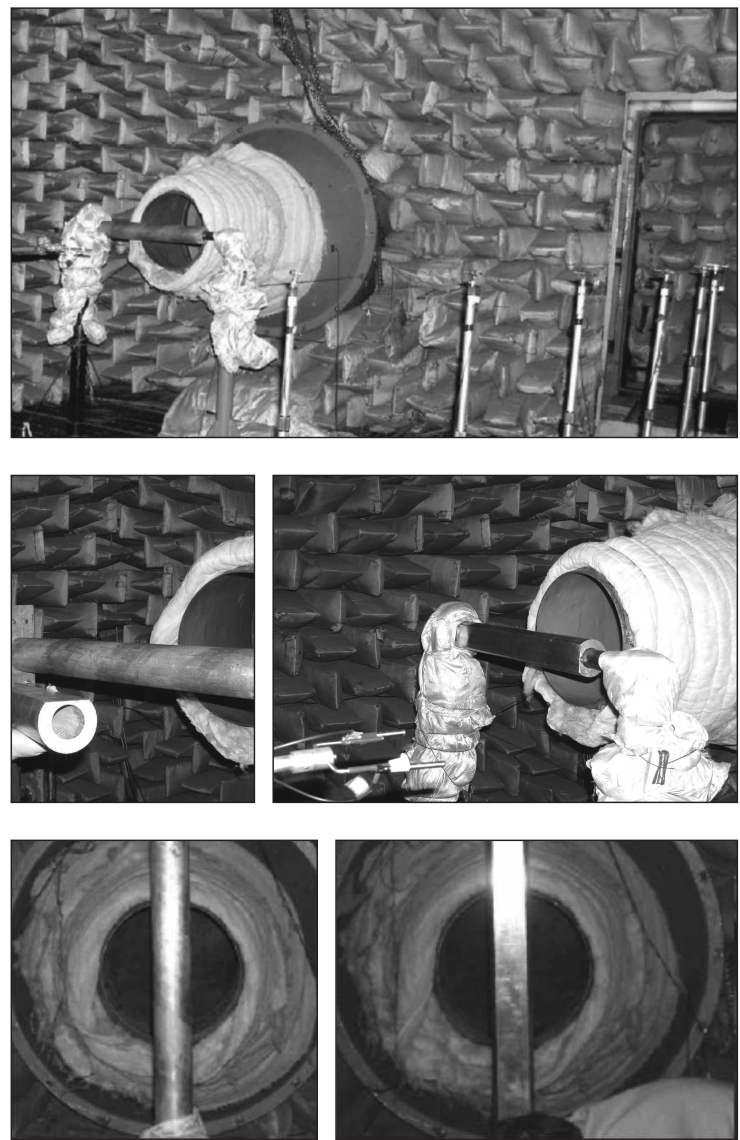

Figure 5 Large-scale cylinder: truncated and round cylinder in anechoic chamber

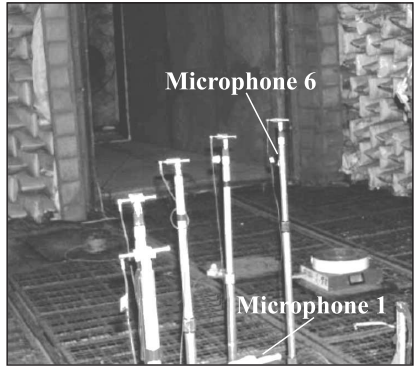

(a)

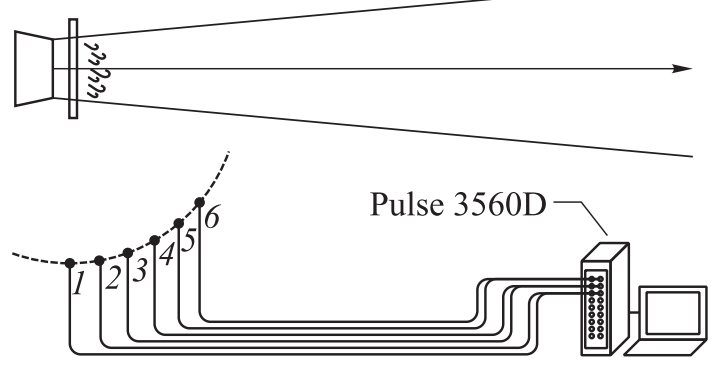

(b)

Figure 6 Polar microphone array $\left(90^{\circ}(1), 80^{\circ}(2), 70^{\circ}(3), 60^{\circ}(4), 50^{\circ}(5)\right.$, and $40^{\circ}(6)$ to flow direction) 

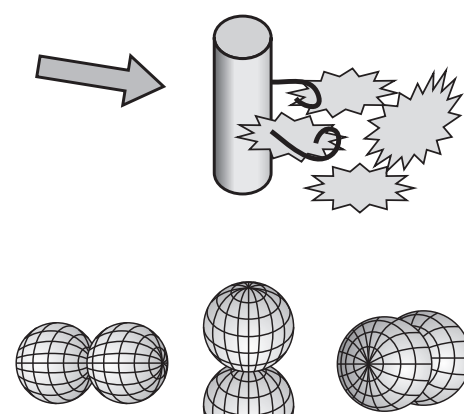

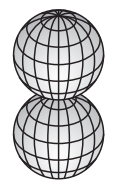

(a)
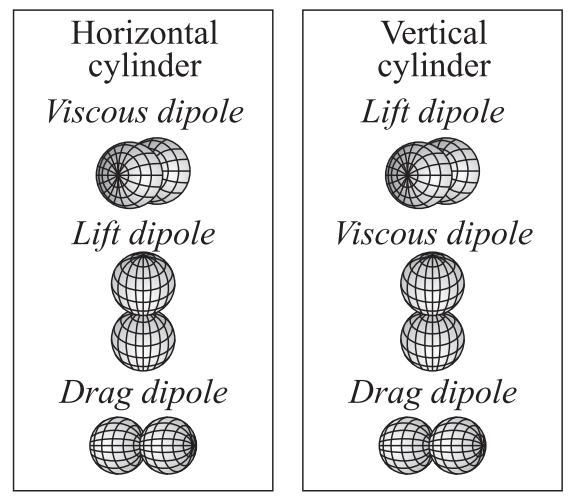

(b)

Figure 7 Directivities of drag, viscous, and lift dipoles

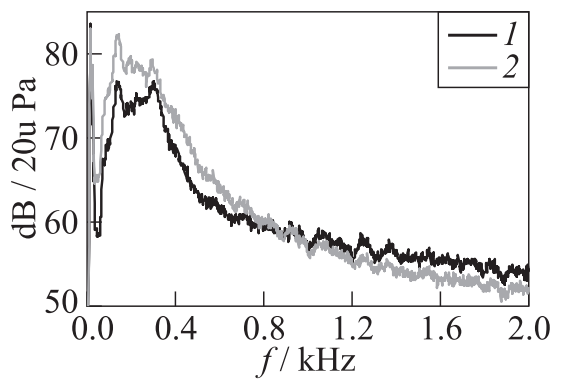

Figure 8 Microphone 1, lift dipole, $\Delta \sim 4 \mathrm{~dB}: 1-$ truncated and $2-$ circular cylinders

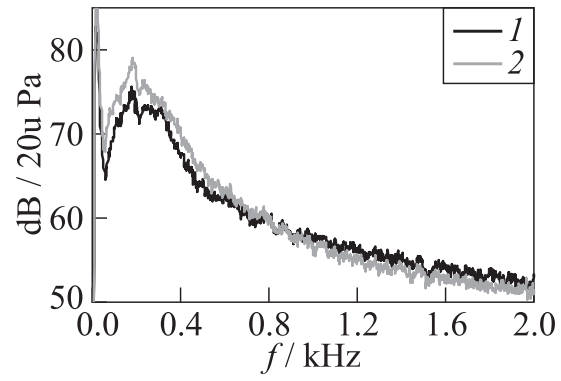

Figure 9 Microphone 6, mix of drag and lift dipoles, $\Delta \sim 2 \mathrm{~dB}: 1-$ truncated and 2 - circular cylinders

$\mathrm{cal} /$ numerical airframe noise prediction models. The applicability of the main concept for noise reduction is considered for large-scale models (Fig. 5). Polar array with microphones (Fig. 6) located in the plane parallel to the floor is used for measurements in far field of dipole components (drag, viscous, and lift dipoles, Fig. 7).

For vertical cylinder, microphone 1 measures only lift dipole (Fig. 8); microphones 2-6 measure mix of lift and drag dipoles (Fig. 9). For horizontal cylinder, microphone 1 measures only viscous dipole (Fig. 10); microphones 2-6 measure mix of lift and drag dipoles (Fig. 11). Amplitude of viscous dipole negligibly small (up to $15 \mathrm{~dB}$ ) in comparison with lift dipole (Fig. 12). 


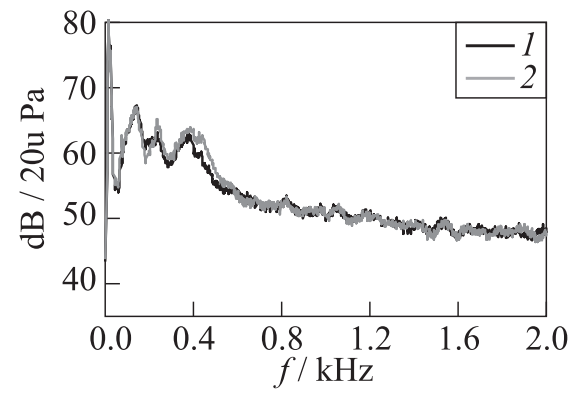

Figure 10 Microphone 1, viscous dipole, $\Delta<1 \mathrm{~dB}: 1$ - truncated and 2 - circular cylinders

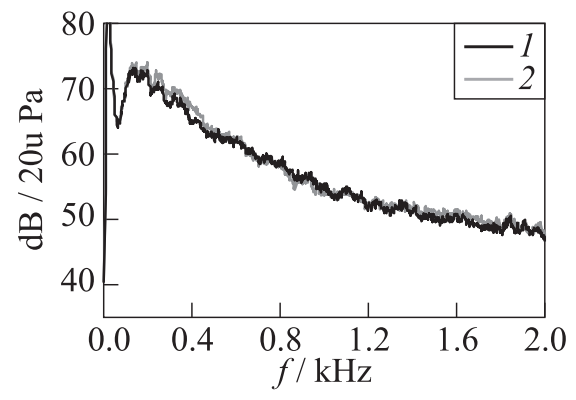

Figure 11 Microphone 6, mix of drag and viscous dipoles, $\Delta \sim 2 \mathrm{~dB}: 1-$ truncated and 2 - circular cylinders

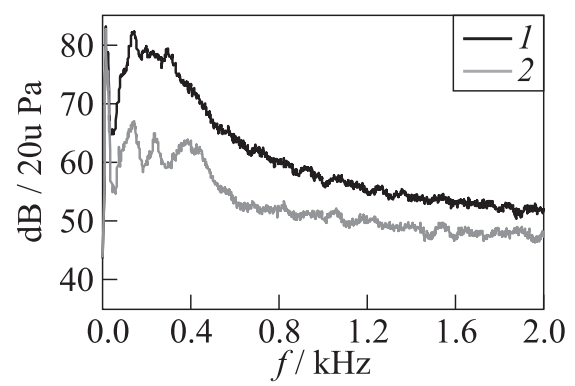

Figure 12 Comparison of lift dipole and viscous dipole amplitudes (microphone 1 data for vertical (1) and horizontal (2) cylinders, respectively), $\Delta \sim 15 \mathrm{~dB}$. Viscous dipole is important only for the angles where lift dipole is absent

\section{CONCLUDING REMARKS}

The effect of noise reduction for truncated geometry is most effective for lift dipole (up to $4 \mathrm{~dB}$ ). To measure the lift dipole by polar array with microphones located in the plane parallel to the floor, one needs to use vertical position of cylinder. Lift dipole is usually dominate in the radiation of bluff cylinders and this type of radiation is attributed as cylinder dipole noise (without splitting the total dipole noise to three orthogonal components). The drag dipole is smaller (at least, in the present experiments) and the viscous dipole (orthogonal to lift and drag dipoles) is negligible. For small-scale cylinder, it is $30 \mathrm{~dB}$ smaller than lift dipole noise. For large-scale model, viscous dipole $15 \mathrm{~dB}$ smaller than lift dipole noise was just obtained; therefore, it would be important only for 
the angles where lift dipole is absolutely absent. Due to this value of orders, even small mistakes (in the orientation of cylinder etc.) could add some input to measurement data; therefore, the spectra peculiarities could be unstable (but due to the small value of the radiation amplitude, these possible mistakes seem to be without any sense). To measure the lift dipole, the cylinder is put in vertical position (shifted $80 \mathrm{~cm}$ downstream the nozzle exit). Thus, the noise reduction for truncated geometry is quite obvious for large Reynolds number (i. e., for large-scale models and high flow velocity).

\section{REFERENCES}

1. Kopiev, V.F., M. Yu. Zaitsev, and R. K. Karavosov. 2004. Experimental investigation of azimuthal structure of dipole noise for rigid cylinder inserted in turbulent jets. AIAA Paper No. 2004-2927.

2. Kopiev, V.F., M. Yu. Zaitsev, and N.N. Ostrikov. 2006. New noise source mechanism of flow/surface interaction as applied to airframe noise reduction. AIAA Paper No. 2006-2717.

3. Terracol, M., and V. Kopiev. 2008. Numerical investigation of the turbulent flow around a truncated cylinder: Noise reduction aspects. AIAA Paper No. 2008-2868. 